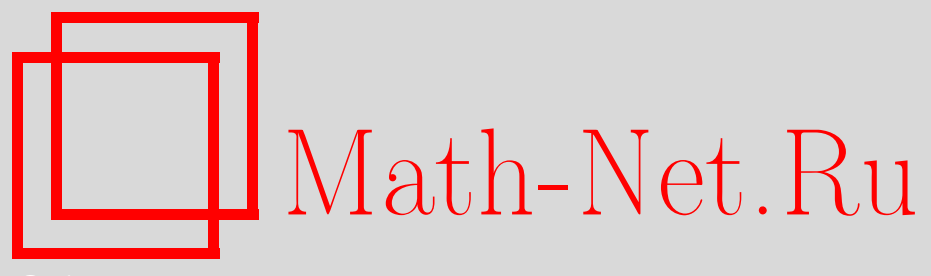

P. В. Михайлов, Асферичность и аппроксимационные свойства скрещенных модулей, Матем. сб., 2007, том 198, номер 4, 79-94

DOI: https://doi.org/10.4213/sm1558

Использование Общероссийского математического портала Math-Net.Ru подразумевает, что вы прочитали и согласны с пользовательским соглашением http://www . mathnet.ru/rus/agreement

Параметры загрузки:

IP: 54.224 .135 .184

26 апреля 2023 г., 15:42:11

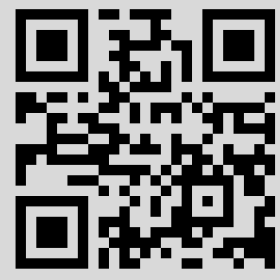




\author{
Р. В. Михайлов
}

\title{
Асферичность и аппроксимационные свойства скрещенных модулей
}

\begin{abstract}
Работа посвящена изучению инвариантов Бэра и аппроксимационных свойств скрещенных модулей и cat $^{1}$-групп. Рассматриваются условия, при которых ядра скрещенных модулей совпадают с пересечением нижнего центрального ряда. Для двумерных комплесов, имеющих асферичную плюс-конструкцию, построен алгебраический критерий асферичности. Как следствие показано, что подкомплекс асферического двумерного комплекса асферичен тогда и только тогда, когда его фундаментальная $\mathrm{cat}^{1}$-группа разрешимо аппроксимируема. Таким образом, дается новая формулировка гипотезы асферичности Уайтхеда в теоретико-групповых терминах.
\end{abstract}

Библиография: 25 названий.

\section{$\S 1$. Введение}

Построение алгебраических моделей гомотопических типов клеточных пространств - одна из основных задач алгебраической топологии. При этом определение "алгебраичности” данной категории также оказывается нетривиальной проблемой. Этой проблеме посвящена интересная работа [1], рассматривающая различные подходы к понятию алгебраической модели категорий гомотопических типов.

Некоторые гомотопические категории имеют естественные алгебраические модели. Гомотопическая категория $\mathscr{H}_{1}$ связных $\mathrm{CW}$-комплексов $K$, для которых гомотопические группы $\pi_{i}(K)$ тривиальны при $i \geqslant 2$, естественным образом эквивалентна категории групп $\mathscr{G}_{r}$. Эквивалентность данных категорий устанавливается с помощью функторов фундаментальной группы и классифицирующего пространства. В случае гомотопической категории $\mathscr{H}_{n} n$-типов, состоящей из связных комплексов $K$, у которых $\pi_{i}(K)=0, i \geqslant n+1$, существует также несколько алгебраических моделей [2]. Неформально говоря, задача построения алгебраических моделей категорий $\mathscr{H}_{n}, n \geqslant 1$, есть задача "алгебраизации" систем Постникова, или задача нахождения "алгебраической гомотопической аппроксимации" данного гомотопического типа. Здесь мы будем рассматривать алгебраические модели категории $\mathscr{H}_{2}$, введенные Дж. Уайтхедом и ЖК.-Л. Лодэем [2]. При этом некоторые результаты настоящей работы могут быть обобщены на алгебраические модели категорий $\mathscr{H}_{n}, n>2$.

Работа выполнена при поддержке Российского фонда фундаментальных исследований (грант № 05-01-00993), а также гранта Президента РФ (№ MK-1487.2005.1). 
Для категории $\mathscr{H}_{2}$ существует несколько удобных алгебраических моделей. Хорошо известно, что следующие категории эквивалентны (см., например, [2]):

- категория $\mathscr{C} \mathscr{M}$ - скрещенных модулей,

- категория $\mathscr{C a t} t^{1}$ cat $^{1}$-групп,

- категория $\mathscr{S} \mathscr{G}{ }_{1}$ - симплициальных групп с комплексом Мура длины $\leqslant 1$,

- категория $\mathscr{C a t}\left(\mathscr{G}_{r}\right)$ - категорных объектов в $\mathscr{G}_{r}$.

Эти категории и оказываются алгебраическими моделями гомотопической категории 2-типов.

Настоящая работа посвящена изучению объектов из категорий $\mathscr{C} \mathscr{M}$ и $\mathscr{C a t} t^{1}$, поэтому приведем определения скрещенных модулей и cat $^{1}$-групп и опустим описание категорий $\mathscr{S}_{1}$ и $\mathscr{C} a t\left(\mathscr{G}_{r}\right)$. Скрещенный модуль есть тройка $(M, \partial, G)$, состоящая из групп $G$ и $M$ таких, что $G$ действует на $M$ (действие мы будем обозначать через $g \circ m$ для $g \in G, m \in M), \partial: M \rightarrow G$ гомоморфизм групп такой, что выполнены следующие условия:

$$
\begin{aligned}
\partial(g \circ m) & =g \partial(m) g^{-1}, & & g \in G, m \in M ; \\
m n m^{-1} & =\partial(m) \circ n, & & m, n \in M .
\end{aligned}
$$

Иногда скрещенный модуль $(M, \partial, G)$ называется $G$-скрещенным модулем. Скрещенный модуль $(M, \partial, G)$ называется асферичным, если ядро $\operatorname{ker}(\partial)$ тривиально.

Пусть $G$ - группа и $s, t \in \operatorname{End}(G)$. Тройка $(G, s, t)$ называется саt ${ }^{1}$-груnпой, если выполнены следующие условия:

1) $s t=t, t s=s$ (под произведением эндоморфизмов подразумевается композиция);

2) $[\operatorname{ker}(s), \operatorname{ker}(t)]=1$.

Функтор $S: \mathscr{C} \mathscr{M} \rightarrow \mathscr{C} a t^{1}$, задающий эквивалентность категорий $\mathscr{C} \mathscr{M}$ и $\mathscr{C} a t^{1}$, может быть задан как

$$
S:(M, \partial, G) \mapsto(M \rtimes G, s:(m, g) \mapsto g, t:(m, g) \mapsto \partial(m) g) .
$$

Для любого комплекса $K$ можно определить фундаментальный скрещенный модуль и фундаментальную саt $^{1}$-группу:

$$
\begin{aligned}
& \mathscr{L}_{1}: K \mapsto\left(\pi_{2}\left(K, K^{(1)}\right), \partial, \pi_{1}\left(K^{(1)}\right)\right), \\
& \mathscr{L}^{1}: K \mapsto \pi_{2}\left(K, K^{(1)}\right) \rtimes \pi_{1}\left(K^{(1)}\right),
\end{aligned}
$$

где $K^{(1)}$ - одномерный остов $K, \partial: \pi_{2}\left(K, K^{(1)}\right) \rightarrow \pi_{1}\left(K^{(1)}\right)$ - гомоморфизм границы. Эндоморфизмы фундаментальной саt ${ }^{1}$-группы $\mathscr{L}^{1}(K)$ естественным образом определяются через гомоморфизм $\partial$.

Д. Кондуше нашел интересную связь между аппроксимационными свойствами некоторых скрещенных модулей и асферичностью гомотопического типа, который они определяют [3]. В частности, он показал, что для подкомплексов асферичных двумерных комплексов второй гомотопический модуль может быть выражен как пересечение нижнего центрального ряда соответствующего скрещенного модуля. Тем самым была дана неожиданная переформулировка 
гипотезы асферичности Уайтхеда, утверждающей асферичность подкомплекса асферического двумерного связного комплекса [3].

Настоящая работа продолжает изучение связи асферичности с аппроксимационными свойствами скрещенных модулей и саt ${ }^{1}$-групп. Используя свойства гомологий и инвариантов Бэра скрещенных модулей, мы получаем (определение нижнего центрального ряда см. ниже)

СлеДСТВИЕ 1. Пусть $(M, \partial, F)$ - скрещенный модуль, для которого группа $F$ свободная, группа $H_{1}(\operatorname{Coker}(\partial))$ свободная абелева и $H_{2} B(M, \partial, F)=0$. Тогда $\operatorname{ker}(\partial)=\gamma_{\omega}(F, M)$.

Используя точность действия коядра на ядре проективного неасферичного модуля, которая доказана в [4], мы также находим связь асферичности некоторых комплексов с разрешимой аппроксимируемостью их фундаментальных cat $^{1}$-групп.

ТеОрема 4. Пусть $K$ - двумерный комплекс, для которого $K^{+}$асферичен. Тогда следующие условия эквивалентнъ:

(i) $\mathfrak{L}^{1}(K)$ разрешимо аппроксимируема;

(ii) $K$ асферичен.

Здесь $K^{+}$- плюс-конструкция Квиллена. При этом имеет место следующий результат Дж. Хаусмана [5]: плюс-конструкция подкомплекса асферичного двумерного комплекса асферична. Таким образом, теорема 4 дает еще одну теоретико-групповую переформулировку гипотезы асферичности Уайтхеда. Эквивалентность асферичности и разрешимой аппроксимируемости фундаментальной cat $^{1}$-группы также имеет место в случае двумерного комплекса, у которого первые гомологии без кручения, а вторые тривиальны (следствие 6).

Помимо упомянутых результатов в настоящей статье мы развиваем методы работы с нижними центральными рядами скрещенных модулей, которые приводят в том числе и к теоретико-групповым результатам.

\section{§ 2. Скрещенные модули}

2.1. Гомологии скрещенных модулей. Классифицирующий функтор

$$
B: \mathscr{C} \mathscr{M} \rightarrow \mathscr{T} p
$$

определен Ж.-Л. Лодэем [2]. Для данного скрещенного модуля $(M, \partial, G)$ пространство $B(M, \partial, G)$ имеет следующие гомотопические группы:

$$
\pi_{1} B(M, \partial, G)=\operatorname{coker}(\partial), \quad \pi_{2} B(M, \partial, G)=\operatorname{ker}(\partial), \quad \pi_{i} B(M, \partial, G)=0, \quad i \geqslant 3,
$$

и обладает свойством, что $\mathscr{L}_{1} B(M, \partial, G)$ слабо эквивалентно $(M, \partial, G)$ в $\mathscr{C} \mathscr{M}$. В работе [2] показано, что этот функтор может быть построен как геометрическая реализация диагонали бинерва $\mathscr{N} \mathscr{N} L(M, \partial, G)$, являющегося бисимплициальным множеством.

Существуют различные пути введения гомологий в рассматриваемых категориях. Например, Г. Эллис определяет гомологии скрещенного модуля $(M, \partial, G)$ 
как гомологии его классифицирующего пространства $H_{*} B(M, \partial, G)[6]$. Это определение удобно с точки зрения вычислений, так как расслоение

$$
K(\operatorname{ker}(\partial), 2) \rightarrow B(M, \partial, G) \rightarrow K(\operatorname{coker}(\partial), 1)
$$

приводит к спектральной последовательности

$$
E_{p, q}^{2}=H_{p}\left(\operatorname{coker}(\partial), H_{q} K(\operatorname{ker}(\partial), 2)\right) \Rightarrow H_{p+q} B(M, \partial, G)
$$

В работе [7] авторы показали, что $\mathscr{C} \mathscr{M}$ является триадной категорией над множествами, и определили гомологии скрещенных модулей в смысле Барра и Бека [8]. ${ }^{1}$ Гомологии, определенные в [7], есть функторы $H_{*}: \mathscr{C} \mathscr{M} \rightarrow \mathscr{A} b(\mathscr{C} \mathscr{M})$ из категории $\mathscr{C} \mathscr{M}$ в категорию $\mathscr{A} b(\mathscr{C} \mathscr{M})$ абелевых скрещенных модулей. Напомним, что скрещенный модуль $(A, \partial, B)$ называется абелевым, если группы $A, B$ абелевы и действие $B$ на $A$ тривиально. Категория абелевых скрещенных модулей оказывается естественным образом эквивалентной категории правых модулей над кольцом нижнетреугольных целочисленных матриц $\left(\begin{array}{ll}\mathbb{Z} & 0 \\ \mathbb{Z} & \mathbb{Z}\end{array}\right)$. Таким

$$
H_{*}(M, \partial, G)=\left(\xi H_{*}(M, \partial, G), h_{*}, k H_{*}(M, \partial, G)\right),
$$

где $\xi H_{*}(M, \partial, G), k H_{*}(M, \partial, G)$ - абелевы группы с тривиальным действием группы $k H_{*}(M, \partial, G)$ на $\xi H_{*}(M, \partial, G)$.

Мы будем использовать следующие свойства гомологий скрещенных модулей.

1) (см. [11]). Для любого скрещенного модуля $(M, \partial, G)$ существует естественная длинная точная последовательность абелевых групп

$$
\cdots \rightarrow H_{n+1} B(M, \partial, G) \rightarrow \xi H_{n}(M, \partial, G) \rightarrow H_{n}(G) \rightarrow H_{n} B(M, \partial, G) \rightarrow \cdots
$$

2) (см. [7]) (5-членная последовательность для скрещенных модулей). Пусть

$$
(N, \mu, R) \rightarrow(Q, \mu, F) \rightarrow(T, \partial, G)
$$

- короткая точная последовательность скрещенных модулей. Тогда существует длинная точная последовательность абелевых скрещенных модулей

$$
\begin{aligned}
H_{2}(Q, \mu, F) & \rightarrow H_{2}(T, \partial, G) \rightarrow\left(\frac{N}{[F, N][R, Q]}, \mu, R /[F, R]\right) \\
& \rightarrow H_{1}(Q, \mu, F) \rightarrow H_{1}(T, \partial, G) \rightarrow 0
\end{aligned}
$$

\footnotetext{
${ }^{1}$ Категории скрещенных модулей являются алгебраическими категориями в смысле Квиллена, поэтому гомологии в этой категории естественно могут быть определены как пучковые гомологии на подходящем сайте Гротендика (см. [9; 5.6]). Это же относится и к гомологиям в категориях $n$-типов, см. [10].
} 
2.2. Инварианты Бэра и нильпотентная аппроксимируемость скрещенных модулей. Определим нижний центральный ряд $\left\{\gamma_{i}(G, M)\right\}_{i \geqslant 1}$ для скрещенного модуля $\partial: M \rightarrow G$ по индукции: $\gamma_{1}(G, M):=M$ и $\gamma_{i+1}(G, M)-$ подгруппа в $M$, порожденная элементами

$$
[g, m]:=(g \circ m) m^{-1}, \quad m \in \gamma_{i}(G, M), \quad g \in G .
$$

Обозначим пересечение подгрупп $\gamma_{i}(G, M), i \geqslant 1$, через $\gamma_{\omega}(G, M)$. Скрещенный модуль $(M, \partial, G)$ назовем нильпотентно аппроксимируемым, если пересечение $\gamma_{\omega}(G, M)=\{1\}$. Также будем использовать стандартные обозначения $\gamma_{i}(G)$, $1 \leqslant i \leqslant \omega$, для нижнего центрального ряда группы $G$ и $[g, h]:=g h g^{-1} h^{-1}$, $g, h \in G$.

Определим инварианты Бэра скрещенного модуля $(M, \partial, G)$ как

$$
B^{(k)}(M, \partial, G)=\operatorname{ker}\left\{\partial_{*}: M / \gamma_{k+1}(G, M) \rightarrow G / \gamma_{k+1}(G)\right\} .
$$

В случае асферичного скрещенного модуля $(R, \partial, F)$ и свободной группы $F$ инварианты Бэра $(R, \partial, F)$ есть классические инварианты Бэра группы $\operatorname{coker}(\partial)$, определяемые как

$$
B^{(k)}(F / R):=\frac{R \cap \gamma_{k}(F)}{\left[R,{ }_{k} F\right]}, \quad k \geqslant 2,
$$

где $\left[R,{ }_{1} F\right]=[R, F],\left[R,{ }_{k+1} F\right]=\left[\left[R,{ }_{k} F\right], F\right]$. Хорошо известно, что в случае группы $G=F / R$ абелева группа $B^{(k)}(G)$ не зависит от выбора $F$ и $R$.

Инварианты Бэра в категории скрещенных модулей, а также более общих категориях интенсивно изучались (см., например, [12], [13]). Обычно инварианты Бэра определяются как производные функторы факторов по аналогам нижнего центрального ряда в данной категории. Наше же определение инвариантов Бэра скрещенных модулей является в некотором смысле примитивным, направленным на прямое обобщение классических инвариантов Бэра для групп.

Для любого скрещенного модуля $(M, \partial, G)$ существует следующая точная последовательность:

$$
H_{2}(G) \rightarrow H_{2}(B(M, \partial, G)) \rightarrow M / \gamma_{2}(G, M) \rightarrow G / \gamma_{2}(G) \rightarrow H_{1}(\operatorname{Coker}(\partial)) \rightarrow 0 .
$$

В частности, если $H_{2}(G)=0$ (например, когда группа $G$ свободная), имеем

$$
B^{(1)}(M, \partial, G)=H_{2} B(M, \partial, G) .
$$

TеОрема 1. Пусть $(M, \partial, F)$ - скрещенный модуль, для которого группа $F$ свободная, $H_{1}(\operatorname{Coker}(\partial))$ - свободная абелева группа и $H_{2} B(M, \partial, F)=0$. Тогда для всех $k \geqslant 1$

$$
B^{(k)}(M, \partial, F)=0 .
$$

ДокАЗАТЕЛЬСтво. Будем доказывать индукцией по $k$.

По условию $B^{(1)}(M, \partial, F)=0$. Пусть $B^{(k)}(M, \partial, F)=0$ для некоторого $k$. Докажем то же самое для $k+1$. Рассмотрим короткую точную последовательность скрещенных модулей:

$$
\left(\gamma_{k}(F, M), \partial_{k}, \gamma_{k}(F)\right) \rightarrow(M, \partial, F) \rightarrow\left(M / \gamma_{k}(F, M), \partial_{k}^{*}, F / \gamma_{k}(F)\right),
$$


где $\partial_{k}^{*}: M / \gamma_{k}(F, M) \rightarrow F / \gamma_{k}(F)$ - гомоморфизм, индуцированный гомоморфизмом $\partial: M \rightarrow F$. Данная последовательность индуцирует следующий эпиморфизм, получающийся из пятичленной последовательности для скрещенных модулей (2):

$$
H_{2}\left(M / \gamma_{k}(F, M), \partial_{k}^{*}, F / \gamma_{k}(F)\right) \rightarrow\left(\gamma_{k}(F, M) / \gamma_{k+1}(F, M), \partial_{k}^{*}, \gamma_{k}(F) / \gamma_{k+1}(F)\right) .
$$

То есть имеем следующую коммутативную диаграмму:

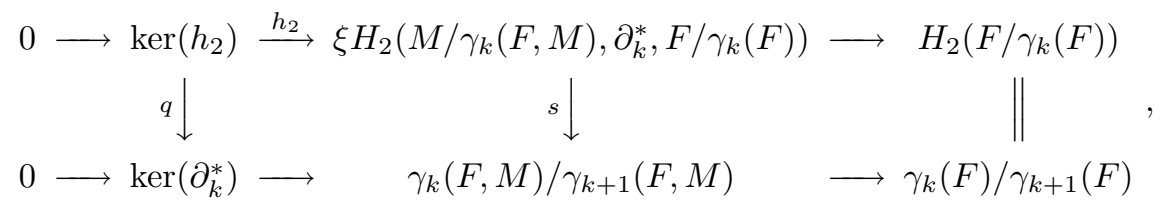

где $s$ - эпиморфизм. Таким образом, $q$ также является эпиморфизмом.

Ввиду точности последовательности

$$
0 \rightarrow \operatorname{ker}\left(\partial_{k}^{*}\right) \rightarrow B^{(k+1)}(M, \partial, F) \rightarrow B^{(k)}(M, \partial, F)
$$

и диаграммы (4) достаточно показать, что $\operatorname{ker}\left(h_{2}\right)=0$. Но благодаря длинной точной последовательности $(1) \operatorname{ker}\left(h_{2}\right)$ можно представить как

$$
\operatorname{ker}\left(h_{2}\right)=\operatorname{coker}\left\{H_{3}\left(F / \gamma_{k}(F)\right) \rightarrow H_{3}\left(B\left(M / \gamma_{k}(F, M), \partial_{k}, F / \gamma_{k}(F)\right)\right)\right\} .
$$

Отметим, что $\operatorname{ker}\left(\partial_{k}\right)=B^{(k)}(M, \partial, F)=0$ по предположению индукции, таким образом,

$$
B\left(M / \gamma_{k}(F, M), \partial_{k}, F / \gamma_{k}(F)\right)=K\left(\operatorname{coker}(\partial) / \gamma_{k}(\operatorname{coker}(\partial)), 1\right)
$$

И

$$
\operatorname{ker}\left(h_{2}\right)=\operatorname{coker}\left\{H_{3}\left(F / \gamma_{k}(F)\right) \rightarrow H_{3}\left(\operatorname{coker}(\partial) / \gamma_{k}(\operatorname{coker}(\partial))\right)\right\}
$$

Tак как $H_{1}(\operatorname{coker}(\partial))$ - свободная абелева группа, то существует подгруппа $H$ в $F$ такая, что ограничение гомоморфизма $\partial$

$$
\left.\partial\right|_{H}: H \rightarrow \operatorname{coker}(\partial)
$$

индуцирует изоморфизм абеленизаций $H / \gamma_{2}(H) \simeq \operatorname{coker}(\partial) / \gamma_{2}(\operatorname{coker}(\partial))$. Отметим, что $H_{2}(\operatorname{coker}(\partial))=0$, так как $H_{2} B(M, \partial, F)$ отображается эпиморфно на $H_{2}(\operatorname{coker}(\partial))$. Следовательно, $\left.\partial\right|_{H}$ индуцирует изоморфизм факторов

$$
H / \gamma_{k}(H) \simeq \operatorname{coker}(\partial) / \gamma_{k}(\operatorname{coker}(\partial))
$$

для всех $k \geqslant 1$. Имеем следующую коммутативную диаграмму:

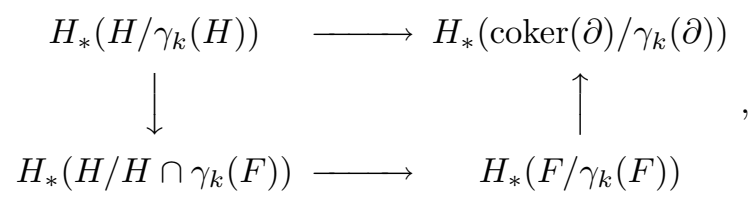

в которой верхний горизонтальный гомоморфизм - изоморфизм. Из диаграммы (6) следует эпиморфность естественного гомоморфизма $H_{*}\left(F / \gamma_{k}(F)\right) \rightarrow$ $H_{*}\left(G / \gamma_{k}(G)\right)$ для всех $k \geqslant 1$ и, следовательно, $\operatorname{ker}\left(h_{2}\right)=0$ ввиду (5). 
СлеДСтвиЕ 1. Пусть $(M, \partial, F)$ - скрещенный модуль, для которого группа $F$ свободная, $H_{1}(\operatorname{coker}(\partial))$ - свободная абелева группа и $H_{2} B(M, \partial, F)=0$. Тогда $\operatorname{ker}(\partial)=\gamma_{\omega}(F, M)$.

ДокАзАТЕЛЬСтво. Очевидно, имеем

$$
\frac{\operatorname{ker}(\partial)}{\operatorname{ker}(\partial) \cap \gamma_{k}(F, M)} \subseteq \operatorname{ker}\left\{M / \gamma_{k}(F, M) \rightarrow F / \gamma_{k}(F)\right\}, \quad k \geqslant 1 .
$$

Из теоремы 1 следует, что $B^{(k)}(M, \partial, F)=0$, т.е. ядро $(7)$ тривиально и $\operatorname{ker}(\partial) \in$ $\gamma_{k}(F, M)$ для всех $k \geqslant 1$. С другой стороны, $\partial\left(\gamma_{k}(F, M)\right) \subseteq \gamma_{k}(F)$. Следовательно, $\partial\left(\gamma_{\omega}(F, M)\right) \subseteq \gamma_{\omega}(F)=1$. Получаем $\gamma_{\omega}(F, M) \subseteq \operatorname{ker}(\partial)$.

Аналогично теореме 1 доказывается следующая

ТеОрема 2. Пусть $(M, \partial, F)$ - скрещенный модуль, для которого группа $F$ свободная и $H_{2} B(M, \partial, F) \otimes \mathbb{Q}=0$. Тогда для всех $k \geqslant 1$

$$
B^{(k)}(M, \partial, F) \otimes \mathbb{Q}=0 .
$$

ДокАЗАТЕЛЬСтво. Доказываем индукцией по $k$.

Предположим, что $B^{(k)}(M, \partial, F) \otimes \mathbb{Q}=0$ для некоторого $k \geqslant 1$. С помощью диаграммы (4) и аргументов, использованных в доказательстве теоремы 1 , легко показать, что для завершения индуктивного шага достаточно установить, что группа

$$
\operatorname{ker}\left(h_{2}\right)=\operatorname{coker}\left\{H_{3}\left(F / \gamma_{k}(F)\right) \rightarrow H_{3} B\left(M / \gamma_{k}(F, M), \partial_{k}^{*}, F / \gamma_{k}(F)\right)\right\}
$$

состоит из элементов конечного порядка.

Расслоение

$$
\begin{aligned}
K\left(B^{(k)}(M, \partial, F), 2\right) & \rightarrow B\left(M / \gamma_{k}(F, M), \partial_{k}^{*}, F / \gamma_{k}(F)\right) \\
& \rightarrow K\left(\operatorname{coker}(\partial) / \gamma_{k}(\operatorname{coker}(\partial)), 1\right)
\end{aligned}
$$

задает гомологическую спектральную последовательность

$$
\begin{aligned}
E_{p, q}^{2}=H_{p}\left(\operatorname{Coker}(\partial) / \gamma_{k}(\operatorname{Coker}(\partial)), H_{q} K\left(B^{(k)}(M, \partial, F), 2\right)\right) & \\
& \Rightarrow H_{p+q} B\left(M / \gamma_{k}(F, M), \partial_{k}^{*}, F / \gamma_{k}(F)\right) .
\end{aligned}
$$

Хорошо известно, что для любой абелевой группы $A$

$$
H_{1} K(A, 2)=0, \quad H_{2} K(A, 2)=A, \quad H_{3} K(A, 2)=0,
$$

следовательно, спектральная последовательность (8) приводит к точной последовательности:

$$
\begin{aligned}
& H_{1}\left(\operatorname{coker}(\partial) / \gamma_{k}(\operatorname{coker}(\partial)), B^{(k)}(M, \partial, F)\right) \rightarrow H_{3} B\left(M / \gamma_{k}(F, M), \partial_{k}^{*}, F / \gamma_{k}(F)\right) \\
& \quad \rightarrow H_{3}\left(\operatorname{coker}(\partial) / \gamma_{k}(\operatorname{coker}(\partial))\right) \rightarrow H_{0}\left(\operatorname{coker}(\partial) / \gamma_{k}(\operatorname{coker}(\partial)), B^{(k)}(M, \partial, F)\right) .
\end{aligned}
$$


По предположению индукции имеем $B^{(k)}(M, \partial, F) \otimes \mathbb{Q}=0$. Следовательно, после тензорного умножения последовательности $(9)$ на $\mathbb{Q}$ получаем естественный изоморфизм

$$
H_{3} B\left(M / \gamma_{k}(F, M), \partial_{k}^{*}, F / \gamma_{k}(F)\right) \otimes \mathbb{Q} \simeq H_{3}\left(\operatorname{coker}(\partial) / \gamma_{k}(\operatorname{coker}(\partial))\right) \otimes \mathbb{Q} .
$$

Таким образом, имеем

$$
\operatorname{ker}\left(h_{2}\right) \otimes \mathbb{Q}=\operatorname{ker}\left\{H_{3}\left(F / \gamma_{3}(F)\right) \rightarrow H_{3}\left(\operatorname{coker}(\partial) / \gamma_{k}(\operatorname{coker}(\partial))\right)\right\} .
$$

Далее действуем как в доказательстве теоремы 1: выбираем подгруппу $H$ в $F$ такую, что имеет место изоморфизм $\mathbb{Q}$-модулей

$$
\left.f\right|_{H}: H \otimes \mathbb{Q} \rightarrow \operatorname{coker}(\partial) \otimes \mathbb{Q}
$$

Применяя теперь $\mathbb{Q}$-версию теоремы Столлингса, получаем, что $\left.f\right|_{H}$ индуцирует изоморфизмы $\mathbb{Q}$-локализаций

$$
H / \gamma_{k}(H) \otimes \mathbb{Q} \simeq \operatorname{coker}(\partial) / \gamma_{k}(\operatorname{coker}(\partial)) \otimes \mathbb{Q} .
$$

Теперь тот же аргумент, что и в доказательстве теоремы 1, приводит к требуемому равенству $\operatorname{ker}\left(h_{2}\right) \otimes \mathbb{Q}=0$.

2.3. Теоретико-групповые приложения. Рассматривая асферичные скрещенные модули, получаем следующие утверждения об инвариантах Бэра.

СлЕДСТВие 2. Пусть $G$ - группа такая, что группа $G_{a b}$ свободная абелева u $\mathrm{H}_{2}(G)=0$. Тогда $B^{(k)}(G)=0, k \geqslant 2$.

СлЕДСТвиЕ 3. Пусть $G$ - группа такая, ито $\mathrm{H}_{2}(G) \otimes \mathbb{Q}=0$.

Тогда $B^{(k)}(G) \otimes \mathbb{Q}=0, k \geqslant 2$.

Следствие 3 доказано в работе [14] с помощью симплициальных методов. Инварианты Бэра играют важную роль при изучении аппроксимационных свойств $k$-центральных расширений групп. Следствие 3 имеет неожиданные приложения. Так, с помощью этого утверждения, в [15] построены примеры нильпотентно аппроксимируемых конечно представленных групп таких, что их $k$ е свободные центральные расширения не являются нильпотентно аппроксимируемыми. Аналогичное приложение находит и следствие 2. Нильпотентно аппроксимируемую группу $G$ называем абсолютно нильпотентно аппроксимируемой, если для любого $k$-центрального расширения

$$
1 \rightarrow N \rightarrow \widetilde{G} \rightarrow G \rightarrow 1
$$

(т.е. $\left[N,{ }_{k} \widetilde{G}\right]=1$ ) группа $\widetilde{G}$ также является нильпотентно аппроксимируемой.

В [15] вводится убывающая фильтрация в инвариантах Бэра, называемая обобщенной фильтрацией Дваера:

$$
B^{(k)}(G)=\varphi_{k+1}^{(k)}(G) \supseteq \varphi_{k+2}^{(k)}(G) \supseteq \cdots, \quad k \geqslant 1, \quad m \geqslant k+1,
$$

определяемая как

$$
\varphi_{m}^{(k)}(G):=\operatorname{ker}\left\{M^{(k)}(G) \rightarrow M^{(k)}\left(G / \gamma_{m-k}(G)\right)\right\} .
$$

Следующее несложное предложение доказано в [15]. 
ПРЕДЛОЖЕНИЕ 1. Пустъ $G$ - әруппа $и \varphi_{m}^{(k)}(G)=0$ для некоторых $k \geqslant 1$ $u m \geqslant k+1$. Для любого $k$-центрального расширения

$$
1 \rightarrow N \rightarrow \widetilde{G} \rightarrow G \rightarrow 1
$$

группа $\widetilde{G}$ нильпотентно аппроксимируема тогда и только тогда, когда нильпотентно аппроксимируема группа $G$.

Следствие 2 и предложение 1 дают

СледСтвиЕ 4. Пусть группа $G$ нильпотентно аппроксимируема, $G_{a b}$ свободная абелева группа, $H_{2}(G)=0$. Тогда $G$ абсолютно нильпотентно аппроксимируема.

Благодаря следствию 4 несложно построить примеры несвободных абсолютно нильпотентно аппроксимируемых групп. Например, группа

$$
G=\langle a, b, c \mid a[b, c][a, c]=1\rangle,
$$

рассмотренная Баумслагом как пример парасвободной группы, является абсолютно нильпотентно аппроксимируемой. Отметим, что остается открытым вопрос: верно ли, что любая группа с одним соотношением, являющаяся нильпотентно аппроксимируемой, также абсолютно нильпотентно аппроксимируема. Оказывается, что любое центральное (и, более того, 2-центральное) расширение группы с одним соотношением нильпотентно аппроксимируемо тогда и только тогда, когда сама группа нильпотентно аппроксимируема [16]. Однако распространить этот результат на произвольные $k$-центральные расширения пока что не удалось.

Отметим, что можно ввести естественный аналог обобщенной фильтрации Дваера для скрещенных модулей. Покажем, как это можно сделать в случае $k=1$, т.е. как обобщить классическую фильтрацию Дваера на случай скрещенных модулей.

Для двух скрещенных $G$-модулей $\partial_{1}: M \rightarrow G, \partial_{2}: N \rightarrow G$ строится скрещенный модуль $\partial_{1} \partial_{2}: M \circ N \rightarrow G$, представляющий собой коамальгаму в категории $G$-скрещенных модулей [17], [18]. При этом имеет место следующая точная последовательность абелевых групп:

$$
\operatorname{ker}\left(\partial_{1}\right) \oplus \operatorname{ker}\left(\partial_{2}\right) \rightarrow \operatorname{ker}\left(\partial_{1} \partial_{2}\right) \rightarrow \frac{R \cap S}{[R, S]} \rightarrow 0
$$

где $R=\operatorname{im}\left(\partial_{1}\right), S=\operatorname{im}\left(\partial_{2}\right)$.

Пусть $\mathscr{M}=(M, \partial, G)$ - скрещенный модуль. Введем следующие обозначения скрещенных модулей:

$$
\mathscr{M}_{2}:=\left(\gamma_{2}(G, M), \partial_{2}, \gamma_{2}(G)\right), \quad \mathscr{G}_{k}:=\left(\gamma_{k}(G), f_{k}, G\right),
$$

где $f_{k}$ - естественное вложение $\gamma_{k}(G) \rightarrow G$.

Определим убывающую фильтрацию

$$
H_{2} B \mathscr{M}=\varphi_{2} H_{2} B \mathscr{M} \supseteq \varphi_{3} H_{2} B \mathscr{M} \supseteq \cdots
$$

как

$$
\varphi_{k} H_{2} B \mathscr{M}:=\operatorname{coker}\left\{\pi_{2} B\left(\mathscr{G}_{k} \circ \mathscr{M}_{2}\right) \rightarrow \pi_{2} B\left(\mathscr{G}_{k} \circ \mathscr{M}\right)\right\}
$$


Тогда из последовательности (11) непосредственно следует

ПредЛОЖЕНИЕ 2. Пусть $\mathscr{M}=(M, \partial, G)$ - скрещенный модуль и $G$ - свободная группа. Тогда для каждого $k \geqslant 2$ имеет место следующая точная последовательность:

$$
\pi_{2} B \mathscr{M} \rightarrow \varphi_{k} H_{2} B \mathscr{M} \rightarrow \varphi_{k}(\operatorname{coker}(\partial)) \rightarrow 1
$$

где $\varphi_{k}(\operatorname{coker}(\partial))=\operatorname{ker}\left\{H_{2}(\operatorname{coker}(\partial)) \rightarrow H_{2}(\operatorname{coker}(\partial)) / \gamma_{k-1}(\operatorname{coker}(\partial))\right\}-\oint$ бильmрация Дваера. В частности, если скрещенный модуль Й коккрофтов, т.е. гомоморфизм Гуревича $\pi_{2} B \mathscr{M} \rightarrow H_{2} B \mathscr{M}$ тривиален, для любого $k \geqslant 2$ имеет место изоморфизм

$$
\varphi_{k} H_{2} B \mathscr{M}=\varphi_{k}(\operatorname{coker}(\partial)) .
$$

2.4. Трансфинитные обобщения. Нижний центральный ряд $\left\{\gamma_{n}(G, M)\right.$, $n \geqslant 1\}$ для скрещенного модуля $(M, \partial, G)$ можно естественным образом продолжить на трансфинитные ординалы, определив $\gamma_{\tau+1}(G, M)$ как подгруппу в $M$, порожденную элементами $[g, m], m \in \gamma_{\tau}(G, M), g \in G$. В случае предельного ординала, как обычно, определяем соответствующий член ряда как пересечение предыдущих.

Последовательности (1) и (2) непосредственно приводят к следующей коммутативной диаграмме:

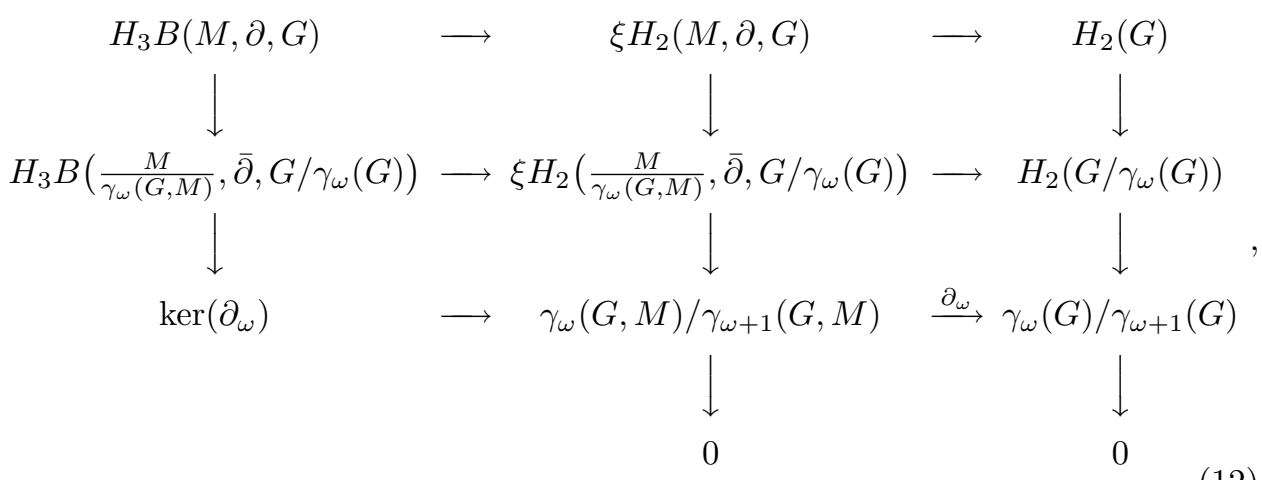

где гомоморфизм $\bar{\partial}: M / \gamma_{\omega}(G, M) \rightarrow G / \gamma_{\omega}(G)$ индуцирован гомоморфизмом $\partial$. В случае свободной группы $G=F$ получаем следующую точную последовательность:

$$
H_{3} B(M, \partial, F) \rightarrow H_{3} B\left(M / \gamma_{\omega}(F, M), \bar{\partial}, F\right) \rightarrow \gamma_{\omega}(F, M) / \gamma_{\omega+1}(F, M) \rightarrow 0 .
$$

В категории $\mathscr{C} \mathscr{M}$ естественным образом определяются проективные объекты (см., например, [18]). При этом имеется теоретико-групповая характеристика проективных скрещенных модулей. В [19] показано, что скрещенный модуль $(M, \partial, G)$ является проективным тогда и только тогда, когда выполнены следующие условия:

1) $M_{a b}$ является проективным $\mathbb{Z}[\operatorname{Coker}(\partial)]$-модулем;

2) гомоморфизм $H_{2}(M) \rightarrow H_{2}(G)$ тривиален.

Из точной последовательности (13) следует 
ПреДЛОжЕНИЕ 3. Пусть $(M, \partial, F)$ - проективный скрещенный модуль, группа $F$ свободна, тогда имеет место следующий изоморфизм:

$$
H_{3} B\left(M / \gamma_{\omega}(F, M), \bar{\partial}, F\right) \simeq \gamma_{\omega}(F, M) / \gamma_{\omega+1}(F, M)
$$

Если к тому же $H_{2} B(M, \partial, F)=0$ и $H_{1}(\operatorname{coker}(\partial))$ - свободная абелева группа, mo

$$
H_{3}(\operatorname{coker}(\partial)) \simeq \gamma_{\omega}(F, M) / \gamma_{\omega+1}(F, M)
$$

ДокАЗАТЕЛЬСтво. В [20] показано, что в случае проективного скрещенного модуля $(M, \partial, F)$ и свободной группы $F$ имеем $H_{3} B(M, \partial, F)=0$. Изоморфизм (14) следует из точности последовательности (13). Пусть теперь $H_{2} B(M, \partial, F)=0$ и $H_{1}(\operatorname{coker}(\partial))$ свободная абелева. Тогда по теореме 1 получаем, что скрещенный модуль $\left(M / \gamma_{\omega}(F, M), \bar{\partial}, F\right)$ aсферичен и $\operatorname{coker}(\partial)=$ $\operatorname{coker}(\bar{\partial})$, откуда следует, что $B\left(M / \gamma_{\omega}(F, M), \bar{\partial}, F\right)$ есть просто $K(\operatorname{coker}(\partial), 1)$ и требуемый изоморфизм следует из (14). Естественно, (15) можно непосредственно получить из теоремы 1 и последовательности Хопфа (последовательности, получаемой из абеленизации скрещенного модуля с последующим применением функтора $\left.H_{0}(\operatorname{coker}(\partial),-)\right)$.

\section{§ 3. Точность действия и аппроксимационные свойства cat $^{1}$-групп}

Пусть $G$ - группа, $M$ - правый $\mathbb{Z}[G]$-модуль. Напомним, что модуль $M$ называется точным, если $\operatorname{Ann}(M):=\{\alpha \in \mathbb{Z}[G] \mid M . \alpha=0\}=0$, и мы говорим, что $G$ действует точно на $M$ (или действие $G$ на $M$ является точным), если $G \cap(1+\operatorname{Ann}(M))=1$. Следующая теорема доказана в [21].

Теорема 3. Пусть $\partial: M \rightarrow F-$ неасферичный скрещенный проективный модуль, F - свободная группа. Тогда стандартное действие coker $(\partial)$ на $\operatorname{ker}(\partial)$ является точным.

Известная теорема Уайтхеда, явившаяся фактически отправной точкой в теории скрещенных модулей, утверждает, что для любого двумерного CW-комплекса $K$ его фундаментальный скрещенный модуль

$$
\partial: \pi_{2}\left(K, K^{(1)}\right) \rightarrow \pi_{1}\left(K^{(1)}\right)
$$

$\left(K^{(1)}\right.$ - одномерный остов $\left.K\right)$ является свободным, а следовательно, проективным скрещенным модулем. Поэтому из теоремы 3 следует, что для любого неасферичного двумерного комплекса $K$ стандартное действие $\pi_{1}(K)$ на $\pi_{2}(K)$ является точным. В случае, когда комплекс $X$ является доминируемым двумерным комплексом, фундаментальный скрещенный модуль (16) также является проективным [19]. Поэтому точность действия $\pi_{1}(K)$ на $\pi_{2}(K)$ имеет место и в случае 2 -доминируемости комплекса $K$. При этом сам модуль $\pi_{2}(K)$, естественно, не обязан быть $\mathbb{Z}\left[\pi_{1}(K)\right]$-точным. Например, в случае проективной плоскости $K=P^{2}$ элемент $1+g$ аннулирует весь модуль $\pi_{2}(K) \simeq \mathbb{Z} \quad(g-$ нетривиальный элемент в $\left.\pi_{1}(K)\right)$.

Напомним, что тройка $\mathscr{M}=(M, \partial, G)$ называется прескрещенным модулем, если выполнено лишь условие (CM1). Скрещенный модуль $\mathscr{M}^{\text {сr }}$ может быть 
получен из $\mathscr{M}$ факторизацией по скобкам Пайффера $\langle c, b\rangle=c b c^{-1} \partial(c) \circ b^{-1}$, $c, b \in M$. В [3] сформулированы следующие гипотезы (см. [3; гипотезы 4.17 и 4.18]). Сильная гипотеза: пусть $\mathscr{M}$ - прескрещенный модуль, у которого $H_{1}(\operatorname{coker}(\partial))$ свободная абелева и $H_{2}(\operatorname{coker}(\partial))=0$, тогда скрещенный модуль $\mathscr{M}^{\text {сr }}$ нильпотентно аппроксимируем. Слабая гипотеза формулируется аналогично, с дополнительным условием $H_{k}(\operatorname{coker}(\partial)), k \geqslant 2$. Из результатов [3] следует, что как слабая, так и сильная гипотеза влекут гипотезу асферичности Уайтхеда. Но теорема 3 позволяет получить простые контрпримеры к этим гипотезам. Пусть $G$ - группа с одним соотношением, не являющаяся нильпотентно аппроксимируемой, у которой $H_{1}(G)$ свободная абелева, $H_{k}(G)=0, k \geqslant 2$. $\mathrm{K}$ примеру, можно взять группу трилистника. Пусть $K$ - некоторый двумерный неасферичный комплекс с фундаментальной группой $G$. Тогда фундаментальный скрещенный модуль $\partial: \pi_{2}\left(K, K^{(1)}\right) \rightarrow \pi_{1}\left(K^{(1)}\right)$ не является нильпотентно аппроксимируемым. Чтобы увидеть это, достаточно заметить, что

$$
(g-1) \circ m \in \gamma_{k}(G, M), \quad m \in \operatorname{ker}(\partial), \quad g \in \gamma_{k}(\operatorname{coker}(\partial)), \quad k \geqslant 2,
$$

для любого скрещенного модуля $(M, \partial, G)$. Детальное доказательство этого факта изложено в [21], хотя надо отметить, что доказательство в [21] не является оптимальным и простейшим. Далее, берем любой элемент $g \in \gamma_{\omega}(G)$ и при предположении нильпотентной аппроксимируемости фундаментального скрещенного модуля $K$ получаем, что $g-1$ аннулирует модуль $\operatorname{ker}(\partial)=\pi_{2}(K)$. Но это противоречит утверждению теоремы 3 . Отсюда следует, что фундаментальный скрещенный модуль комплекса $K$ не является нильпотентно аппроксимируемым, что дает контрпримеры к вышеупомянутым гипотезам.

Ниже мы будем использовать аналогичный аргумент, но для производного ряда в группах и сat ${ }^{1}$-группах. Для группы $G$, как обычно, через $\delta_{n}(G)$ обозначается $n$-й член производного ряда $G, \delta_{\omega}=\bigcap_{n} \delta_{n}(G), \delta_{\omega+1}(G)=\left[\delta_{\omega}(G), \delta_{\omega}(G)\right]$. Группа $G$ называется разрешимо аппроксимируемой, если $\delta_{\omega}(G)=1$. Через $\mathscr{P}(G)$ будем обозначать совершенный радикал группы $G$, т.е. наибольшую максимальную подгруппу в $G$, равную своему коммутанту. Ниже мы также будем использовать термин "разрешимая аппроксимируемость" и в отношении $\mathrm{cat}^{1}$-групп. При этом будем говорить, что сat ${ }^{1}$-группа $(G, s, t)$ paзреuимо anпроксимируема, если разрешимо аппроксимируема группа $G$.

Для данного комплекса $K$ через $K^{+}$обозначается плюс-конструкция $K$. Напомним, что комплекс $K^{+}$получается из комплекса $K$ приклеиванием двумерных и трехмерных клеток и универсален относительно свойства тривиальности совершенного радикала $\mathscr{P}\left(\pi_{1}\left(K^{+}\right)\right)$фундаментальной группы $\pi_{1}\left(K^{+}\right)$. При этом фундаментальная группа $K^{+}$оказывается изоморфной фактору $\pi_{1}(K) / \mathscr{P}\left(\pi_{1}(K)\right)$.

ТеОрема 4. Пусть $K$ - двумерный комплекс, для которого $K^{+}$асферичен. Тогда следующие условия эквивалентны:

(i) $\mathfrak{L}^{1}(K)$ разрешимо аппроксимируема;

(ii) $K$ асферичен. 
ДоказАтельство. Тот факт, что (ii) влечет (i), очевиден. Действительно, если комплекс $K$ асферичен, имеем точную последовательность групп:

$$
1 \rightarrow \pi_{2}\left(K, K^{(1)}\right) \rightarrow \pi_{1}\left(K^{(1)}\right) \rightarrow \pi_{1}(K) \rightarrow 1,
$$

т.е. группа $\pi_{2}\left(K, K^{(1)}\right)$ является подгруппой свободной группы $\pi_{1}\left(K^{(1)}\right)$. Обозначим $R=\pi_{2}\left(K, K^{(1)}\right), F=\pi_{1}\left(K^{(1)}\right)$. Тогда, очевидно,

$$
\gamma_{\omega}\left(\mathscr{L}^{1}(K)\right)=\gamma_{\omega}(R \rtimes F)=\bigcap_{k \geqslant 2}\left[R,{ }_{k} F\right]=1,
$$

т.е. группа $R \rtimes F$ нильпотентно аппроксимируема. Поэтому (ii) влечет (i). Условие (i), очевидно, выполняется для любого асферичного комплекса $K$.

Теперь предположим, что группа $\mathscr{L}^{1}(K)$ разрешимо аппроксимируема, а комплекс $K$ не является асферичным. Из того, что плюс-конструкция $K^{+}$ асферична, следует, что $\pi_{1}(K)$ имеет нетривиальный совершенный радикал $\mathscr{P}\left(\pi_{1}(K)\right)$. Таким образом, можно найти нетривиальный элемент $x \in \delta_{n}\left(\pi_{1}(K)\right)$ для всех $n \geqslant 1$.

Заметим, что для любого скрещенного модуля $\partial: M \rightarrow P$ имеет место включение

$$
(p-1) \circ m \in \delta_{n}(M \rtimes P), \quad p \in \delta_{n}(P), \quad m \in M,
$$

ввиду того, что $(x-1) \circ m=[m, x]$. Следовательно,

$$
(x-1) \circ m \in \delta_{n}\left(\pi_{2}\left(K, K^{(1)}\right) \rtimes \pi_{1}\left(K^{(1)}\right)\right), \quad m \in \pi_{2}(K), \quad n \geqslant 1 .
$$

Разрешимая аппроксимируемость группы $\mathscr{L}^{1}(K)$ влечет, что элемент $x-1$ аннулирует весь модуль $\pi_{2}(K)$, но это противоречит точности действия $\pi_{1}(K)$ на $\pi_{2}(K)$ (теорема 3 ). Поэтому $\pi_{2}(K)=0$ и условие (i) влечет условие (ii).

Условие об асферичности плюс-конструкции возникает достаточно естественно в ряде случаев. В работе [22] показано, что для данного двумерного комплекса $K$ следующие условия эквивалентны:

(i) $K^{+}$асферичен;

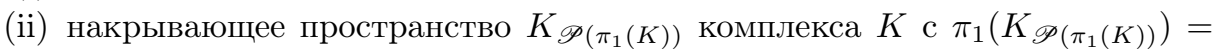
$\mathscr{P}\left(\pi_{1}(K)\right)$ ациклично;

(iii) $H_{2}\left(\mathscr{P}\left(\pi_{1}(K)\right)\right)=0$ и $K$ является $\mathscr{P}\left(\pi_{1}(K)\right)$-коккрофтовым комплексом. ${ }^{2}$

В частности, для любого подкомплекса $K$ асферичного двумерного комплекса $L$ плюс-конструкция $K^{+}$асферична [5]. Отсюда следует, что гипотеза асферичности Уайтхеда, утверждающая, что любой подкомплекс связного асферичного двумерного комплекса асферичен, может быть переформулирована в теоретико-групповых терминах.

\footnotetext{
${ }^{2}$ Напомним, что для группы $G$, нормальной подгруппы $H$ в $G$ и $G$-модуля $M$, вложимого в свободный $G$-модуль $f: M \rightarrow \mathbb{Z}[G]^{\oplus \alpha}$, говорят, что $M$ является $H$-коккрофтовым, если композиция $M \rightarrow \mathbb{Z}[G]^{\oplus \alpha} \rightarrow \mathbb{Z}[G / H]^{\oplus \alpha}$ тривиальна, где последнее отображение индуцировано эпиморфизмом $G \rightarrow G / H$. В случае, когда $M=\pi_{2}(K)$ - второй гомотопический модуль двумерного комплекса $K$ и $G=\pi_{1}(K), H$-коккрофтовость эквивалентна тривиальности гомоморфизма Гуревича $H$-накрытия $K_{H}$ комплекса $K$.
} 
СледСтвиЕ 5. Пусть $L$ - асферичный двумерный комплекс, $K$ - подкомплекс L. Тогда следующие условия эквивалентны:

(i) комплекс $K$ асферичен;

(ii) бундаментальная cat $^{1}$-группа $\mathscr{L}^{1}(K)$ разрешимо аппроксимируема.

Следуя методам работ [23] и [24], можно получить гомологические условия, обеспечивающие эквивалентность асферичности и разрешимой аппроксимируемости фундаментальной cat $^{1}$-группы.

СледСтвиЕ 6. Пусть $K$ - двумерный комплекс, у которого $H_{1}(K)$ без кручения и $\mathrm{H}_{2}(K)=0$. Тогда следующие условия эквивалентны:

(i) $\mathfrak{L}^{1}(K)$ разрешимо аппроксимируема;

(ii) $K$ асферичен.

Доказательство следует схеме доказательства теоремы 4.3 из [24].

Группа $G$ называется E-группой (см. [23]), если

1) абеленизация $G_{a b}$ не содержит кручения,

2) существует $\mathbb{Z}[G]$-резольвента над $\mathbb{Z}$

$$
P_{2} \stackrel{\partial_{2}}{\rightarrow} P_{1} \rightarrow P_{0} \rightarrow \mathbb{Z} \rightarrow 0
$$

такая, что гомоморфизм

$$
1 \otimes \partial_{2}: \mathbb{Z} \otimes_{\mathbb{Z}[G]} P_{2} \rightarrow \mathbb{Z} \otimes_{\mathbb{Z}[G]} P_{1}
$$

является мономорфизмом.

В [23] показано, что совершенный радикал $\mathscr{P}(G) E$-группы $G$ также является $E$-группой. Более того, в качестве $\mathbb{Z}[\mathscr{P}(G)]$-резольвенты можно взять $\mathbb{Z}[G]$-резольвенту такую, что $1 \otimes \partial_{2}$ - мономорфизм, при этом $1 \otimes_{\mathbb{Z}[\mathscr{P}(G)]} \partial_{2}$ также будет мономорфизмом.

В [24] показано (лемма 4.1, случай двумерного комплекса), что если $K-$ двумерный комплекс, $N$ - подгруппа в $\pi_{1}(K)$, то следующие условия эквивалентны:

(i) $1 \otimes_{N} \partial_{2}: \mathbb{Z} \otimes_{\mathbb{Z}[N]} C_{2} \widetilde{K} \rightarrow \mathbb{Z} \otimes_{\mathbb{Z}[N]} C_{1} \widetilde{K}$ является мономорфизмом, где $C_{*} \widetilde{K}$ - цепной комплекс универсального накрытия $\widetilde{K}$ над $K$;

(ii) $K$ является $N$-коккрофтовым и $H_{2}(N)=0$.

Таким образом, для двумерного комплекса $K$, у которого $H_{1}(K)$ без кручения и $H_{2}(K)=0$, получаем, что $\pi_{1}(K)$ является $E$-группой. Поэтому $\mathscr{P}\left(\pi_{1}(K)\right)$ также является $E$-группой и, более того, гомоморфизм

$$
1 \otimes_{\mathscr{P}\left(\pi_{1}(K)\right)} \partial_{2}: \mathbb{Z} \otimes_{\mathbb{Z}\left[\mathscr{P}\left(\pi_{1}(K)\right)\right]} C_{2} \widetilde{K} \rightarrow \mathbb{Z} \otimes_{\mathbb{Z}\left[\mathscr{P}\left(\pi_{1}(K)\right)\right]} C_{1} \widetilde{K}
$$

является мономорфизмом. Снова применяя лемму 4.1 из [24], получаем, что $K$ является $\mathscr{P}\left(\pi_{1}(K)\right)$-коккрофтовым. Отсюда следует по упомянутым условиям асферичности плюс-конструкции, что $K^{+}$асферичен. Теперь следствие 6 вытекает из теоремы 4.

Отметим, что для любого скрещенного модуля $\partial: M \rightarrow P$ со свободной группой $P$ группа $M$ нильпотентно аппроксимируема. Это следует из того, 
что группа $M$ является центральным расширением свободной группы. Так как группа $P$ нильпотентно аппроксимируема, получаем, что $2 \omega$-член трансфинитного производного ряда $\delta_{2 \omega}$ полупрямого произведения $M \rtimes P$ тривиален. Таким образом, стабилизация трансфинитного производного ряда группы $M \rtimes P: \delta_{\omega}(M \rtimes P)=\delta_{\omega+1}(M \rtimes P)$ влечет разрешимую аппроксимируемость группы $M \rtimes P$. Получаем следующее

ЗАмЕчАниЕ 1. В формулировке теоремы 4 условие (i) эквивалентно стабилизации трансфинитного производного ряда фундаментальной cat $^{1}$-группы $\mathscr{L}^{1}(K)$ :

$$
\delta_{\omega}\left(\mathscr{L}^{1}(K)\right)=\delta_{\omega+1}\left(\mathscr{L}^{1}(K)\right) .
$$

В заключение отметим, что методы, использующие точность действия, как правило, не позволяют описать второй гомотопический модуль, как это сделано, например, в следствии 1. Возникает следующий

Вопрос. Пусть $K$ - двумерный комплекс, у которого $K^{+}$асферичен. Верно ли, что $\pi_{2}(K)=\delta_{\omega}\left(\mathscr{L}^{1}(K)\right)$ ?

Автор благодарит Р. Брауна, Д. Кондуше и И. Б. С. Пасси за стимулирующие обсуждения и ценные замечания. Некоторые из результатов настоящей работы содержатся в препринте [25], подготовленном автором во время визита в IHES (Bures-sur-Ivette, France).

\section{Список литературы}

[1] J. Rubio, F. Sergeraert, "Algebraic models for homotopy types", Homology Homotopy Appl., 7:2 (2005), 139-160.

[2] J.-L. Loday, "Spaces with finitely many non-trivial homotopy groups", J. Pure Appl. Algebra, 24:2 (1982), 179-202.

[3] D. Conduché, "Question de Whitehead et modules précroisés", Bull. Soc. Math. France, 124:3 (1996), 401-423.

[4] Р. В. Михайлов, “Точные действия групп и асферические комплексы”, Геометрическая топология, дискретная геометрия и теория множеств, Тр. МИАН, 252, 2006, 184-193; англ. пер.: R. V. Mikhailov, "Faithful group actions and aspherical complexes", Proc. Steklov Inst. Math., 252 (2006), 172-181.

[5] J.-C. Hausmann, Acyclic maps and the Whitehead aspherical problem, Preprint.

[6] G. J. Ellis, "Homotopy of 2-types", J. London Math. Soc., 46:1 (1992), 1-27.

[7] P. Carrasco, A. M. Cegarra, A. R.-Grandjeán, "(Co)Homology of crossed modules", J. Pure Appl. Algebra, 168:2-3 (2002), 147-176.

[8] M. Barr, J. Beck, "Homology and standard constructions", Seminar on triples and categorical homology theory (ETH, Zürich, 1966/67), Lecture Notes in Math., 80, Springer-Verlag, Berlin, 1969, 245-335.

[9] D. G. Quillen, Homotopical algebra, Lecture Notes in Math., 43, Springer-Verlag, Berlin-Heidelberg-New York, 1967.

[10] J. M. Casas, G. Ellis, M. Ladra, T. Pirashvili, "Derived functors and the homology of n-types", J. Algebra, 256:2 (2002), 583-598.

[11] A. R. Grandjean, M. Ladra, T. Pirashvili, "CCG-homology of crossed modules via classifying spaces", J. Algebra, 229:2 (2000), 660-665.

[12] T. Everaert, T. Van der Linden, "Baer invariants in semi-abelian categories II: Homology", Theory Appl. Categ., 12:4 (2004), 195-224. 
[13] A. Frohlich, "Baer-invariants of algebras", Trans. Amer. Math. Soc., 109:2 (1963), 221-244.

[14] G. Ellis, "A Magnus-Witt type isomorphism for non-free groups", Georgian Math. J., 9:4 (2002), 703-708.

[15] Р.В. Михайлов, "Инварианты Бэра и нильпотентная аппроксимируемость групп”, Изв. РАН. Сер. матем., 71:2 (2007), 151-172.

[16] Р.В. Михайлов, "О нильпотентной и разрешимой аппроксимируемости групп", Матем. сб., 196:11 (2005), 109-126; англ. пер.: R. V. Mikhailov, "Residual nilpotence and residual solubility of groups", Sb. Math., 196:11 (2005), 1659-1675.

[17] R. Brown, "Coproducts of crossed P-modules: Applications to second homotopy groups and to the homology of groups", Topology, 23:3 (1984), 337-345.

[18] M. N. Dyer, "Crossed modules and $\pi_{2}$ homotopy modules", Two-dimensional homotopy and combinatorial group theory, London Math. Soc. Lecture Note Ser., 197, Cambridge Univ. Press, Cambridge, 1993, 125-156.

[19] J. G. Ratcliffe, "Free and projective crossed modules", J. London Math. Soc., 22:1 (1980), 66-74.

[20] T. Datuashvili, T. Pirashvili, "On (co)homology of 2-types and crossed modules", J. Algebra, 244:1 (2001), 352-365.

[21] R. Mikhailov, "On residual properties of projective crossed modules", Comm. Algebra, 34:4 (2006), 1451-1458.

[22] N.D. Gilbert, "Cockroft complexes and the plus construction", Groups-Korea'94, Proceedings of the international conference (Pusan, Korea, 1994), eds. A. C. Kim et al., Gruyter, Berlin, 1995, 119-125.

[23] R. Strebel, "Homological methods applied to the derived series of groups", Comment. Math. Helv., 49 (1974), 302-332.

[24] J. Brandenburg, M. Dyer, "On J. H. C. Whitehead's aspherical question I", Comment. Math. Helv., 56:1 (1981), 431-446.

[25] R. Mikhailov, On obstructions to asphericity of certain crossed modules, Preprint: IHES/M/05/45.

P. В. Михайлов (R. V. Mikhařlov)

Математический институт

им. В. А. Стеклова РАН

E-mail: rmikhailov@mail.ru
Поступила в редакцию 18.04.2006 и 28.11.2006 